\title{
Premenstrual Psychosis In A Patient With Polycystic Ovary Syndrome: A Case Report
}

\section{Tamara Pang ( $\nabla$ tamara.pang@mohh.com.sg )}

National University Hospital https://orcid.org/0000-0001-7901-5704

Yit Shiang Lui

National University Hospital

\section{Tji Tjian Chee}

National University Hospital

\section{Case report}

Keywords: Periodic, psychosis, menstrual cycle, premenstrual, female, estrogen

Posted Date: June 30th, 2020

DOI: https://doi.org/10.21203/rs.3.rs-38120/v1

License: (c) (1) This work is licensed under a Creative Commons Attribution 4.0 International License. Read Full License 


\title{
Premenstrual Psychosis In A Patient With Polycystic Ovary Syndrome: A Case Report
}

Tamara Pang ${ }^{1}$, Yit Shiang, Lui ${ }^{1}$, Chee Tji Tjian ${ }^{1}$

${ }^{1}$ Department of Psychological Medicine, National University Hospital, Singapore

Corresponding Author: Tamara Pang, tamara.pang@mohh.com.sg

\begin{abstract}
Periodic psychosis in relation to the menstrual cycle phases has been reported but its pathogenesis not yet known. We report a case of a 28 year old female with schizoaffective disorder since 18 years old. She was diagnosed with polycystic ovary syndrome (PCOS) 3 years later and was noted to have distinct psychotic episodes that coincided acutely with her premenstrual period. Peculiarly she exhibited delusions of pregnancy during her psychotic episodes. This was followed by near resolution with the end of menstruation. This case sheds light on this atypical presentation of psychosis in female patients as well as its potential aetiology.
\end{abstract}

\section{Keywords}

Periodic, psychosis, menstrual cycle, premenstrual, female, estrogen

\section{Background}

Periodic psychosis related to the menstrual cycle was described as early as the mid$19^{\text {th }}$ century by the French psychiatrist, Brière de Boismont, who reported 4 cases of premenstrual insanity $[1,2]$. This unique presentation of psychosis was further expounded upon by von Krafft-Ebbing in 1902 [3], and later by Jolly in 1914 [4] who developed a temporal classification of the disorder in relation to the stage of reproductive life [5]. Since then, there have been between 80 to 280 reported cases worldwide [6, 7]. More recently, Brockington has further refined the subclassification of menstrual psychoses by timing within the menstrual cycle [6]: 
1. Premenstrual: psychosis starts during the second half of the cycle, and sometimes ends with abrupt recovery at the onset of menstrual bleeding

2. Catamenial: psychoses which begin with the onset of menstrual flow

3. Paramenstrual: psychosis occurs at variable timing, always in harmony with the menstrual cycle

4. Mid-cycle: onset almost mid-way between the beginning of menstrual bleeding

5. Epochal: bipolar psychoses lasting for the complete cycle, with switches linked to menstruation

These cases raise the possibility of a hormonal role in the manifestation of psychosis in a subgroup of female patients. Despite such works, this concept remains relatively unrecognised and forgotten. Particularly in Singapore, there have been no reported cases of menstrual psychosis. A literature review revealed only 2 cases of menstrual psychosis in association with PCOS reported worldwide $[8,9]$. We report a case of periodic psychosis associated with the premenstrual phase of the menstrual cycle presenting with prominent delusions of pregnancy in a patient with PCOS. Through this case, the authors hope to explore a possible aetiology behind premenstrual psychosis in relation to PCOS, with a secondary aim to explain our patient's delusions of pregnancy.

\section{Case presentation}

A 28 year old Chinese female was admitted to an inpatient psychiatric facility after presenting to the hospital's emergency department with the intention to deliver her unborn child. She was convinced that she has 5 months pregnant and cited the symptoms of abdominal pain and missed menstruation. She had a known diagnosis of schizoaffective disorder since the age of 18 with 6 previous psychiatric admissions during which she had exhibited similar features of delusions of pregnancy. Her past medical history was characterised by Type 2 diabetes mellitus, PCOS and obesity. Prior to admission, she was on olanzapine $15 \mathrm{mg}$ ON and aripiprazole $20 \mathrm{mg}$ OM. 
During the admission, she reported her last menstrual period to be 5 months ago and held the belief that she had been impregnated by a celebrity. Both urine and serum beta-hCG were negative. Corroborative history from her parents revealed a distinct pattern, in which the patient exhibited episodes of psychosis that coincided with her premenstrual period. The exacerbation of her psychotic symptoms came on in the few days leading up to her menses with gradual resolution as menstrual bleeding ceased. In between these episodes, in the intermenstrual period, she remained relatively well, with no overt positive symptoms of psychosis. She continued to have meaningful friendships and engaged in social activities. However she had shown an overall decline in function over the 10 years since her diagnosis, demonstrated by her inability to sustain employment and dependence on her mother to administer her insulin.

A review of her past psychotropics showed that she had fared poorly on risperidone and had experienced hyperprolactinaemia as a side effect of the medication leading to oligomenorrhoea. Risperidone was ceased. Since 2017, she has remained relatively stable on a combination of olanzapine and aripiprazole.

Within a few days of admission, and with gradual uptitration of olanzapine from $15 \mathrm{mg}$ to $20 \mathrm{mg} \mathrm{ON}$, she no longer held the belief that she was pregnant and had achieved euthymia. She was discharged well and at baseline with close outpatient follow-up.

\section{Discussion}

An underlying hormonal aetiology in relation to PCOS in premenstrual psychosis

Estrogen has been hypothesised to exert a protective effect against psychosis [10]. Supporting this, conditions associated with estrogen withdrawal and hypoestrogenic states have been reported to precipitate or exacerbate psychotic symptoms [11]. This has been demonstrated by various reports of acute psychosis with withdrawal of estrogen such as cases of psychosis occuring post-abortion, with cessation of 
exogenous estrogen (e.g. oral contraceptive pills) and administration of estrogen receptor antagonists (e.g. clomiphene, Tamoxifen) [12].

Estrogen is known to have a neuromodulatory effect on the central nervous system (CNS) and has been shown to block dopamine receptors [13]. A period of high and sustained estrogen levels, as with anovulatory cycles, leads to the upregulation of dopaminergic receptors and hence increased sensitivity. This has been demonstrated in studies using both human and animal models, that showed increased dopaminergic receptor sensitivity in the luteal phase of the menstrual cycle $[14,15]$. The subsequent estrogen cascade that happens in the luteal phase preceding menstruation can lead to dopaminergic overactivity giving rise to psychotic symptoms. [16]

PCOS is characterised by anovulatory cycles, during which there is relatively unopposed estrogenic stimulation, priming the CNS for a premenstrual fall in estrogen at the next ovulatory period [9]. This pathogenetic mechanism may explain the clinical picture of our patient with PCOS, who exhibits a clear exacerbation of her psychotic symptoms in the premenstrual phase.

Early treatments for such cases of periodic psychoses associated with the menstrual cycle were aimed at interrupting menstruation via means like ovariectomy and artificial induction of menopause [5]. However these proved to be unsuccessful. Conventional psychotropics have also been demonstrated to be inadequate in interrupting the periodic nature of this illness [7]. More recent literature has reported the successful use of alternative treatment strategies targeting the hypothalamic-pituitary-ovarian axis [17]. These include hormone replacement, in the form of combined oral contraceptive pills [18] or a GnRH agonist [19], and metformin, in the setting of PCOS to restore normal ovulation [8].

\section{Possible theories behind the delusions of pregnancy}

A secondary finding in our patient was the manifestation of her psychotic symptoms in the form of delusions of pregnancy. It is our hypothesis that antipsychotic-induced 
metabolic syndrome, hyperprolactinaemia and high estrogen levels in the early luteal phase each played a part in her delusion of pregnancy by causing symptoms akin to the somatic experience of pregnancy.

Hyperprolactinaemia is a known side effect of most first generation and some second generation antipsychotics. The high estrogen levels in the anovulatory cycles of PCOS also lead to dopamine blockade and a consequent increase in prolactin, compounding the effect of antipsychotic use. This resulting hperprolactinaemia may serve to trigger or strengthen delusional beliefs of pregnancy through its symptoms of galactorrhoea and amenorrhoea [20]. There have been various cases in the literature documenting a temporal association between hyperprolactinaemia and delusions of pregnancy that resolved with normalisation of prolactin levels and change in antipsychotic agent [21].

Antipsychotic-induced metabolic syndrome also gives rise to features that may further fuel this delusion, such as widening abdominal girth and breast tenderness/engorgement [22]. The high estrogen levels in the early luteal phase of the menstrual cycle similarly result in such effects on the body.

Therefore a combination of adverse effects from antipsychotic use and elevated estrogen levels, gives rise to bodily symptoms that together may precipitate and reinforce delusions of pregnancy by mimicking its symptoms.

In our patient, she suffered from obesity which was likely multifactorial in nature, including PCOS, type 2 diabetes mellitus and antipsychotic-induced weight gain. Her oligomenorrhoea was likely secondary to PCOS and possibly hyperprolactinaemia. She was treated with olanzapine and aripirazole after being unable to tolerate risperidone due to hyperprolactinaemia in the past.

\section{Limitations}

The main limitation of this case is its retrospective nature. We were unable to determine the exact dates in relation to her menstrual cycle during which psychotic 
symptoms emerged. This was partly due to the unreliable nature of the menstrual history provided by a patient in an actively psychotic state. In addition, we were not able to obtain blood investigations to document serum hormonal levels during the acute episode, such as those of estrogen and prolactin, to further evaluate the underlying pathogenesis. Althought our patient's acute psychotic exacerbation resolved with uptitration of olanzapine, there may be a need to consider alternative treatment in the form of hormonal therapy to prevent her cyclilcal relapses, while simultaneously bearing in mind potential adverse effects in a young female of childbearing age.

\section{Conclusion}

This case illustrates the complexity of mental illness in women's health. It is important for clinicians to elicit psychotic symptoms in relation to the menstrual cycle in female patients. Patients can also be encouraged to monitor their symptoms via menstrual diaries. More emphasis should be placed on perimenstrual psychoses and we hope to encourage further research into the possible underlying hormonal aetiology, which can in turn provide evidence for treatment strategies other than conventional psychotropics. Considering the potential role of anovulatory cycles and estrogen in perimenstrual psychoses, psychiatrists should also be aware of the use of antipsychotics that may exacerbate anovulation via hyperprolactinaemia.

\section{List of abbreviations}

PCOS: Polycystic ovary syndrome

CNS: Central nervous system

\section{Declarations}

\section{Ethics approval and consent to participate}

Not applicable 


\section{Consent for publication}

Informed consent was obtained from the patient and her mother for publication of this case report.

Availability of data and materials

Not applicable

\section{Competing interests}

The authors declare that they have no competing interests.

Funding

Not applicable

Author's contributions

All authors contributed to, read and approved the final manuscript.

Acknowledgments

Not applicable

\section{References}

1. de Boismont AJFB. De la menstruation: considérée dans ses rapports physiologiques et pathologiques: Baillière; 1842.

2. Brière de Boismont $A$. Recherches bibliographiques et cliniques sur la folie puerpérale, précédées d'un aperçu sur les rapports de la menstruation et de l'aliénation mentale. Ann Méd-Psychol. 1851;3:574-610.

3. von Krafft-Ebing R. Psychosis menstrualis: eine klinisch-forensische Studie: Enke; 1902.

4. Jolly P. Menstruation und psychose. Archiv für Psychiatrie und Nervenkrankheiten. 1915;55(3):637-86.

5. Brockington I. Menstrual psychosis. Archives of Women's Mental Health. 1998;1(1):3-13. 
6. BROCKINGTON I. Menstrual psychosis. World Psychiatry. 2005;4(1):9.

7. Brockington IF. Menstrual psychosis: a bipolar disorder with a link to the hypothalamus. Current psychiatry reports. 2011;13(3):193.

8. Andreou C, Syngelakis M, Karavatos A. Metformine for psychosis associated with the menstrual cycle in a patient with polycystic ovary syndrome. Archives of women's mental health. 2008;11(5-6):387.

9. Jalnapurkar I, Findley JC. A case of repeated mixed mood episodes with psychotic symptoms associated with the premenstrual period in a patient with polycystic ovarian syndrome. Gynecological Endocrinology. 2018;34(6):467-9.

10. Seeman MV, Lang M. The role of estrogens in schizophrenia gender differences. Schizophrenia Bulletin. 1990;16(2):185-94.

11. Seeman M. Menstrual exacerbation of schizophrenia symptoms. Acta psychiatrica scandinavica. 2012;125(5):363-71.

12. Mahe V, Dumaine A. Oestrogen withdrawal associated psychoses. Acta Psychiatrica Scandinavica. 2001;104(5):323-31.

13. McEwen BS, Alves SE. Estrogen actions in the central nervous system. Endocrine reviews. 1999;20(3):279-307.

14. Wieck A, Davies R, Hirst A, Brown N, Papadopoulos A, Marks M, et al. Menstrual cycle effects on hypothalamic dopamine receptor function in women with a history of puerperal bipolar disorder. Journal of psychopharmacology. 2003;17(2):204-9.

15. Czoty PW, Riddick NV, Gage HD, Sandridge M, Nader SH, Garg S, et al. Effect of menstrual cycle phase on dopamine D2 receptor availability in female cynomolgus monkeys. Neuropsychopharmacology. 2009;34(3):548-54.

16. Deuchar N, Brockington I. Puerperal and menstrual psychoses: the proposal of a unitary etiological hypothesis. Journal of Psychosomatic Obstetrics \& Gynecology. 1998;19(2):104-10.

17. Ward HB, Greenberg JA, Almeida M. Perimenstrual psychiatric hospitalization: case report and literature review. Archives of women's mental health. 2019:1-7.

18. Sadurni MC, Rodie JU, de Montagut LM, Autet MS. The use of oral contraceptives as a prevention of recurrent premenstrual psychosis. Psychiatry research. 2009;170(2-3):290-1. 
19. Heinzman JT, Buckingham ET. Menstrual Psychosis and the Workup of New-Onset Psychosis in a Teenager. JAACAP Connect.36.

20. Ali JA, Desai KD, Ali LJ. Delusions of pregnancy associated with increased prolactin concentrations produced by antipsychotic treatment. International Journal of Neuropsychopharmacology. 2003;6(2):111-5.

21. Ahuja N, Vasudev K, Lloyd A. Hyperprolactinemia and delusion of pregnancy. Psychopathology. 2008;41(1):65-8.

22. Manjunatha N, Saddichha S. Delusion of pregnancy associated with antipsychotic induced metabolic syndrome. The World Journal of Biological Psychiatry. 2009;10(4-2):669-70. 